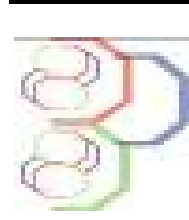

Journal of Applied Biosciences 124: 12416-12423

\title{
Analyse comparée des effets de deux amendements organiques sur le statut organo-minéral et la productivité d'un sol sableux
}

\author{
Ferdinand Gohi Bi ZRO1', Dogniméton SORO1 et Damien Hebert Akré ABOBI ${ }^{1}$ \\ 1 Université Jean Lorougnon Guédé, UFR Agroforesterie, BP 150 Daloa, Côte d'Ivoire ; \\ 2Université Félix Houphouët-Boigny, UFR Sciences de la Terre et des Ressources Minières, 22 BP 582 Abidjan 22, \\ Côte d'Ivoire. \\ Auteur correspondant : Ferdinand Gohi Bi ZRO ; E-mail : zraubigof@yahoo.com ; Tél : (+225) 48713491.
}

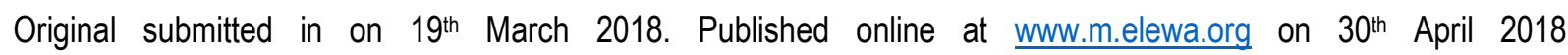
https://dx.doi.org/10.4314/jab.v124i1.3

\section{RESUMÉ}

Objectif : La présente étude vise à déterminer et comparer l'influence du fumier de bovin et du compost d'ordures ménagères sur le statut organo-minéral d'un sol sableux utilisé en maraîchage à Daloa (Côte d'Ivoire).

Méthodologie et résultats : Un essai agronomique portant sur l'espèce de laitue Lactuca sativa a été mis en place. Cet essai constitué de blocs aléatoires complets répétés trois fois consistait en trois traitements : un témoin ou sol sans amendement, un apport au sol de 40 t/ha, d'une part de fumier de bovin, et d'autre part de compost d'ordures ménagères. La valeur fertilisante des amendements a été déterminée par des analyses de laboratoire effectuées sur leurs échantillons. L'effet induit sur le sol par les amendements a été déterminé par l'analyse d'échantillons de sol provenant des différents traitements. En outre, la biomasse fraîche totale produite par chaque traitement a été déterminée. Les résultats ont montré une augmentation du stock de matières organiques dans les sols amendés, notamment le sol ayant reçu le fumier. Par conséquent, la CEC, les niveaux de phosphore, potassium, magnésium, calcium et sodium de ces sols ainsi que leur productivité ont été améliorés significativement.

Conclusion et application des résultats : Le fumier de bovin ayant présenté une valeur fertilisante des sols plus intéressante et permis une meilleure productivité du sol étudié peut être recommandé à la dose de 40 t/ha pour la fertilisation des sols sableux.

Mots clés : Amendement organique, Statut organo-minéral, Productivité, Sol sableux.

Comparative analysis of the effects of two organic amendments on the organo-mineral status and productivity of a sandy soil

ABSTRACT

Objectives: The present study aims to determine and compare the influence of cattle manure and waste compost on the organo-mineral status of a sandy soil used for market gardening in Daloa (Côte d'Ivoire).

Methodology and results : An agronomic test on lettuce species Lactuca sativa has been put in place. This trial consisting of complete randomized blocks repeated three times consisted of three treatments : a control or unmodified soil and a feed of $40 \mathrm{t} / \mathrm{ha}$, on the one hand, of cattle manure, and on the other hand, of compost 
household waste. The fertilizing value of the amendments was determined by laboratory analyzes carried out on their samples. The effect induced on the soil by the amendments was determined by the analysis of soil samples taken from the different treatments. In addition, the total fresh biomass produced by each treatment was determined. The results showed an increase in the stock of organic matter in the amended soils. As a result, their levels of CEC, phosphorus, potassium, magnesium, calcium, sodium and their productivity have been significantly improved.

Conclusion and applications of results: For having presented a more interesting soils fertilizer value in general and allowed a better productivity of the soil studied, cattle manure can be recommended at the feed of 40 t/ha to the fertilization of sandy soils.

Keywords: Organic amendment, Organo-mineral status, Productivity, Sandy soil.

\section{INTRODUCTION}

En Afrique, la croissance démographique dans les grandes villes a engendré une augmentation de la demande alimentaire qui ne peut être satisfaite que par une intensification de l'activité agricole (Milleville and Serpante, 1994). Ainsi, l'Afrique Subsaharienne doit augmenter sa production agricole de $4 \%$ chaque année pour répondre à cette demande (Griffon et al., 1993). Or, la croissance démogra phique s'accompagne toujours d'une diminution des terres cultivables et, par conséquent, d'une pression accrue sur ces terres. II en résulte que la fertilité des sols agricoles diminue, et avec elle, les rendements agricoles. Cette situation non reluisante de l'agriculture en Afrique n'est toutefois pas irrémédiable. En effet, Pour Jouve (2011), en Afrique de l'Ouest par exemple, la production céréalière pourrait largement doubler si on donnait aux agriculteurs les moyens techniques et financiers d'utiliser des engrais chimiques et organiques. Ces engrais, notamment les engrais chimiques, sont

\section{MATÉRIELS ET MÉTHODES}

Cadre physique de la zone d'étude: Le périmètre d'étude, à savoir, la ville de Daloa couvre une superficie de $54,33 \mathrm{~km}^{2}$. Cet espace est situé au centre ouest de la Côte d'Ivoire, pays de l'Afrique de l'ouest ; il est délimité par les longitudes Ouest $6,48^{\circ}$ et $6,41^{\circ}$ et les latitudes Nord $6,91^{\circ}$ et $6,84^{\circ}$ (Figure 1). Le régime climatique de la région du Haut-Sassandra dont Daloa est le Chef-lieu est celui du domaine Guinéen caractérisé par un régime équatorial et subéquatorial à deux maxima pluvio métriques (Brou, 2005). Le relief y est peu contrasté, peu varié et dominé par des plateaux de 200 à $400 \mathrm{~m}$ d'altitude (Avenard, 1971). Les formations géologiques qui couvrent la région administrative du Haut-Sassandra sont celles du Précambrien moyen dominées encore hors de portée de la plupart des producteurs, faute de l'insuffisance des structures d'appui à l'agriculture (Jouve, 2011 ; Fondio et al., 2011). Ainsi , les déjections d'animaux et/ou les composts qui sont très disponibles et de moindres coûts sont de plus en plus utilisés par les producteurs de maraîchers pour amender leurs sols, qui sont en général des sols sableux (Temgoua et al., 2017; Zro al., 2017 et 2018). Toutefois, la variation significative des rendements agricoles obtenus lorsque les producteurs appliquent l'un ou l'autre des amendements organiques, suscite la curiosité pour la détermination de la valeur fertilisante de ces amendements organiques. La présente étude s'inscrit dans cette démarche. Elle vise à déterminer et comparer l'influence sur le statut organo-minéral d'un sol sableux du fumier de bovin et du compost d'ordures ménagères, deux amendements organ iques utilisés régulièrement par les producteurs de maraichers de la ville de Daloa en Côte d'Ivoire.

essentiellement par les granites. Les sols de la zone sont en général ferralitiques moyennement lessivés sur terres fermes et hydromorphes sableux sur les terrasses des rivières (Dabin et al., 1960). Ces sols sableux sont plus ou moins utilisables suivant leur texture, leur richesse chimique et leur possibilité d'irrigation ou de drainage (Dabin et al., 1960). A Aaloa, on les rencontre dans tous les secteurs de la ville, notamment aux quartiers Commerce et Labia (centre ville), Lobia (nord-ouest), Tazibouo (nord), Corridor d'Issia (sud) et Huberson (ouest), où ils sont utilisés régulièrement pour le maraîchage. L'étude a été réalisée sur l'un de ces sols sableux, dans un bas-fond localisé au quartier Tazibouo (Figure 1). Selon Zro et al. (2016), le taux de sables dans 
ce sol dépasse les $80 \%$ quand son $\mathrm{pH}$ moyen avoisine 5,1 . Ses teneurs moyennes en carbone organique et en azote total sont respectivement de 0,71 et 0,45 \%. Concernant le phosphore assimilable et les bases échangeables, à savoir, le calcium, le magnésium, le potassium et le sodium ces teneurs moyennes sont estimées respectivement à : $0,02 \mathrm{~g}_{\mathrm{kg}} \mathrm{kg}^{-1}, 0,22,0,16,0,4$ et $0,10 \mathrm{cmol}^{\mathrm{kg}} \mathrm{g}^{-1}$.

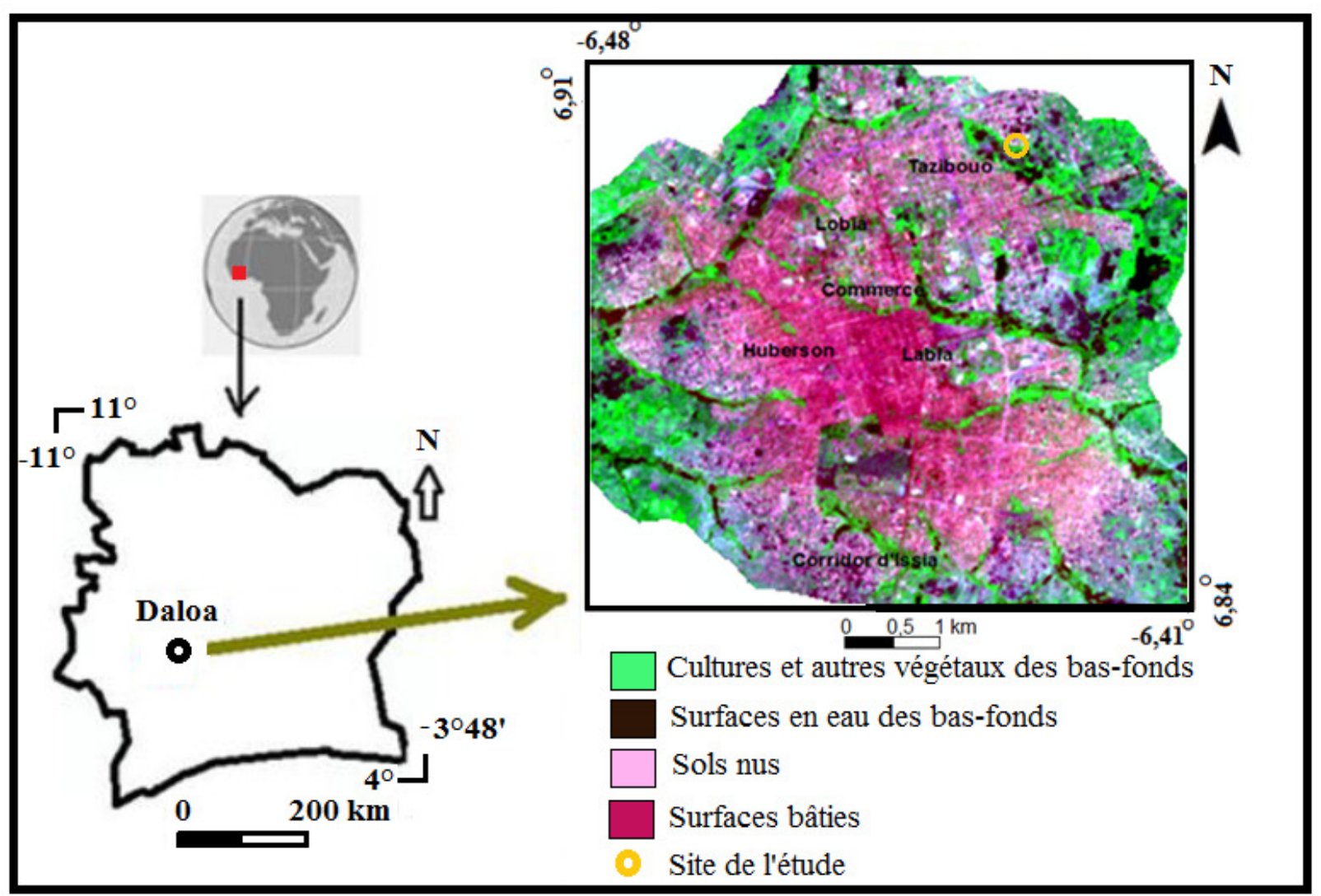

Figure 1 : Carte de localisation de la zone d'étude.

Matériels : Trois types de matériel ont été utilisés pour réaliser cette étude. L'espèce Lactuca sativa a été utilisée comme matériel végétal, en raison de son cycle court. Ainsi, cette plante peut permettre, en un temps record, plusieurs séries d'observations et mesures, en vue de porter sur une parcelle expérimentale, un diagnostic précis des facteurs limitants de la production ou de l'efficacité des intrants. Aussi, un matériel fertilisant constitué de fumier de bovin et de compost d'ordures ménagères a-t-il été utilisé. Le fumier provenait d'une ferme d'élevage située à environ $500 \mathrm{~m}$ de la parcelle expérimentale quand le compost a été prélevé dans une décharge publique d'ordures située à moins de $300 \mathrm{~m}$ du site de l'étude. Le dernier type de matériel utilisé est un matériel technique. II est constitué d'outils de mesures des distances et/ou des longueurs (un mètre ruban et un double décimètre), de défrichage (machettes), de sarclage (dabas) et de mesure des masses (balance Roberval de capacité $15 \mathrm{~kg}$ ).

\section{Méthodes :}

Mise en place de l'essai : L'essai agronomique mis en place est constitué de blocs aléatoires complets répétés trois fois. Cet essai comportait trois traitements : un témoin (sol sans amendement) et deux traitements d'apports de $40 \mathrm{t} /$ ha de fumier de bovin d'une part et de compost d'ordures ménagères d'autre part. Chaque bloc est long de $15 \mathrm{~m}$ et large de $4 \mathrm{~m}$. La distance inter-bloc mesure $2 \mathrm{~m}$. La parcelle élémentaire est une planche de $9 \mathrm{~m}^{2}(4,5 \mathrm{~m} \times 2 \mathrm{~m})$ sur laquelle 16 plants de laitue ont été repiqués sur trois lignes avec un écartement de $30 \mathrm{~cm}$ entre les plants. Les lignes étaient séparées aussi de 30 $\mathrm{cm}$. Quarante huit poquets ont été ainsi obtenus par parcelle élémentaire, soit 53333 poquets à l'hectare. Le repiquage des plants sur les parcelles élémentaires a été effectué après une mise en pépinière de 15 jours. Durant 
tout leur cycle de développement, les plants ont été régulièrement arrosés chaque jour, matin et soir.

Collecte des données : Les propriétés fertilisantes des amendements étudiés ont été déterminées suite à des analyses de laboratoire effectuées sur leurs échantillons. L'effet de ces amendements sur le sol a été déterminé également par des mesures de laboratoire effectuées sur des échantillons de sol des parcelles élémentaires. Aussi, la biomasse fraîche totale produite par traitement a-t-elle été déterminée. L'échantillonnage des fertilisants a été réalisé selon les prescriptions existantes en la matière (Chabalier et al., 2006). II s'est agi de constituer un mélange et un brassage de plusieurs prélèvements et choisir de manière aléatoire, les échantillons à analyser. Ainsi, quatre échantillons de chaque amendement ont été retenus. Ces échantillons ont été emballés dans des sachets plastiques et conservés au frais. Au laboratoire, le $\mathrm{pH}$ a été déterminé par la méthode électrométrique (CEAEQ, 2005), les teneurs en azote total, carbone organique, phosphore assimilable et bases échang eables, respectivement par les techniques de Kjeldahl, Walkley et Black, Olsen modifiée et de spectro photométrie d'absorption atomique (Pétard, 1993). L'échantillonnage des sols a été réalisé environ 15 mois à partir de la date de la première application des

\section{RÉSULTATS}

Caractéristiques des amendements organiques utilisés : Les caractéristiques du fumier de bovin et du compost utilisés sont présentées dans le tableau 1. Leurs teneurs en azote ne sont pas très différentes $(P>0,05)$, ce qui n'est pas le cas du carbone qui est plus abondant dans le fumier $(P<0,05)$. De même, le rapport $\mathrm{C} / \mathrm{N}$ du fumier de bovin est significativement plus élevé que celui du compost $(P<0,05)$. Le pH illustre que le compost est amendements, après trois cycles successifs de la culture de laitue mise en place. Les cycles étaient intercalés chaque fois d'un mois, temps observé pour préparer le sol à la prochaine culture. Le prélèvement a été fait dans la couche arable du sol ( 0 à $20 \mathrm{~cm}$ de profondeur) sur chaque catégorie de parcelle élémentaire. Ainsi, 16 échantillons de sol ont été prélevés au total en vue des analyses chimiques. Dans le cadre de ces analyses, les variables déterminées et les méthodes employées étaient identiques à celles déterminées et effectuées pour le cas des amendements. La biomasse fraîche totale des laitues produites par traitement a été mesurée à la fin de chaque cycle de culture. $\bar{A}$ cet effet, les laitues ont été entièrement déterrées, classées par traitement et pesées in situ à l'aide de la balance Roberval utilisée. Analyse des données : Les valeurs moyennes des variables du sol et celles des biomasses mesurées ont été soumises à une analyse de variance ANOVA à un facteur à l'aide du logiciel Statistica 7.0 (Statsoft, Tulsa, USA). Le test post hoc LSD de Fisher a été réalisé pour constituer les groupes homogènes. Pour la comparaison des propriétés des amendements, le test $t$ de Student au seuil de $5 \%$ a été réalisé pour faire la comparaison deux à deux des moyennes.

faiblement alcalin alors que le fumier de bovin l'est fortement. Outre le sodium dont le niveau est apparu presqu'identique dans les deux amendements, les niveaux du phosphore assimilable, du calcium, du magnésium et du potassium sont différents de manière très hautement significative $(P<0,001)$. Hormis le calcium, le fumier de bovin renferme les teneurs les plus élevées de ces éléments.

Tableau 1 : Propriétés des amendements utilisés

\begin{tabular}{|c|c|c|c|c|c|c|c|c|c|}
\hline & \multicolumn{3}{|c|}{ Statut organique } & \multirow{2}{*}{$\begin{array}{l}\text { Acidité } \\
\text { pHeau }\end{array}$} & \multicolumn{5}{|c|}{ Statut minéral } \\
\hline & $\begin{array}{c}C \\
(\%)\end{array}$ & $\begin{array}{c}N \\
(\%)\end{array}$ & $\mathrm{C} / \mathrm{N}$ & & $\begin{array}{l}\text { P.ass } \\
\left(\text { g. }^{-1}{ }^{-1}\right)\end{array}$ & $\begin{array}{c}\mathrm{Ca}^{2+} \\
\left(\mathrm{cmol}^{2} \mathrm{k}\right. \\
\left.\mathrm{g}^{-1}\right)\end{array}$ & \begin{tabular}{|c}
$\mathrm{Mg}^{2+}$ \\
$\left(\mathrm{cmol}^{2} \mathrm{k}\right.$ \\
$\left.\mathrm{g}^{-1}\right)$ \\
\end{tabular} & $\begin{array}{c}\mathrm{K}^{+} \\
(\mathbf{c m o l} . \mathrm{k} \\
\left.\mathrm{g}^{-1}\right) \\
\end{array}$ & $\begin{array}{c}\mathrm{Na}^{+} \\
\left(\mathrm{cmol}^{-\mathrm{k}}\right. \\
\left.\mathrm{g}^{-1}\right)\end{array}$ \\
\hline $\begin{array}{l}\text { Fumier } \\
\text { de } \\
\text { bovin }\end{array}$ & $\begin{array}{l}13,15 \pm 0, \\
54 a\end{array}$ & $\begin{array}{l}1,10 \pm 0,0 \\
6 \mathbf{a}\end{array}$ & $\begin{array}{l}11,93 \pm 1 \\
03 a\end{array}$ & $\begin{array}{l}9,38 \pm 0,1 \\
1 \mathbf{a}\end{array}$ & $\begin{array}{l}0,17 \pm 0,0 \\
2 a\end{array}$ & $\begin{array}{l}7,51 \pm 0,1 \\
5 \mathbf{a}\end{array}$ & $\begin{array}{l}2,98 \pm 0,0 \\
3 \mathbf{a}\end{array}$ & $\begin{array}{l}2,40 \pm 0,1 \\
3 a\end{array}$ & $\begin{array}{l}0,13 \pm 0,0 \\
2 a\end{array}$ \\
\hline $\begin{array}{l}\text { Comp } \\
\text { ost }\end{array}$ & $\begin{array}{l}10,06 \pm 0, \\
39 \mathrm{~b}\end{array}$ & $\begin{array}{l}1,12 \pm 0,0 \\
4 a\end{array}$ & $9 \pm 0,60 \mathrm{~b}$ & $\begin{array}{l}7,30 \pm 0,1 \\
4 \mathbf{b}\end{array}$ & $\begin{array}{l}0,06 \pm 0,0 \\
0 \mathbf{b}\end{array}$ & $\begin{array}{l}8,75 \pm 0,0 \\
3 \mathbf{b}\end{array}$ & $\begin{array}{l}2,40 \pm 0,0 \\
5 b\end{array}$ & $\begin{array}{l}0,97 \pm 0,0 \\
5 \mathbf{b}\end{array}$ & $\begin{array}{l}0,17 \pm 0,0 \\
1 \mathrm{a}\end{array}$ \\
\hline Test $t$ & 6,580 & 5,660 & 5,88 & 133,34 & 43,09 & 64,51 & 94,22 & 108,12 & 2,01 \\
\hline$P$ & 0,041 & 0,054 & 0,048 & 0,0000 & 0,0005 & 0,0001 & 0,0000 & 0,0000 & 0,205 \\
\hline
\end{tabular}


Effets des amendements organiques utilisés sur le statut organo-minéral du sol : II ressort des résultats figurant dans le tableau 2 que l'apport des amendements organiques au sol à la dose de 40 t/ha a impacté de manière très hautement significative les propriétés du sol. En effet, les teneurs en carbone et en azote du sol ainsi que le ratio $(\mathrm{C} / \mathrm{N})$ de ces deux éléments ont augmenté de manière très hautement significative $(P<0,001)$. Le fumier de bovin a induit un effet plus important sur les teneurs alors que le compost s'illustrait mieux au niveau du ratio. Les résultats montrent également que le niveau du phosphore assimilable a été relevé légèrement $(P>$ $0,05)$ quand ceux du potassium, magnésium, calcium et sodium, puis la CEC augmentaient de manière très hautement significative $(P<0,001)$. Pour chacune de ces variables du sol, excepté le phosphore assimilable et le sodium, les valeurs les plus élevées ont été observées sur les sols amendés avec le fumier. Par ailleurs, le pH du sol, qui était acide $(5,15)$ tend vers la neutralité avec le fumier de bovin $(6,5)$ en passant par un niveau faiblement acide $(6,2)$ avec le compost. Cette hausse de l'acidité du sol est avérée hautement significative $(P<0,001)$.

Tableau 2 : Propriétés des sols en fin d'expérimentation

\begin{tabular}{|c|c|c|c|c|c|c|c|c|c|c|}
\hline & \multicolumn{3}{|c|}{ Statut organique } & \multirow{2}{*}{$\begin{array}{l}\text { Acidité } \\
\text { pHeau }\end{array}$} & \multicolumn{6}{|c|}{ Statut minéral } \\
\hline & $\begin{array}{c}C \\
(\%)\end{array}$ & $\begin{array}{c}N \\
(\%)\end{array}$ & $\mathrm{C} / \mathrm{N}$ & & $\begin{array}{l}\text { P.ass } \\
\left(\text { g.kg-1) }^{-1}\right)\end{array}$ & $\begin{array}{l}\text { CEC } \\
\text { (cmol. } \\
\left.\mathrm{kg}^{-1}\right)\end{array}$ & $\begin{array}{c}\mathrm{Ca} \\
\left(\mathrm{cmol}^{-} .\right. \\
\left.\mathrm{kg}^{-1}\right)\end{array}$ & $\begin{array}{c}\mathrm{Mg} \\
(\mathrm{cmol} . \\
\left.\mathrm{kg}^{-1}\right)\end{array}$ & $\begin{array}{c}\mathrm{K} \\
\text { (cmol.kg } \\
1 \text { ) }\end{array}$ & $\begin{array}{c}\mathrm{Na} \\
\left(\mathrm{cmol}^{-\mathrm{k}}\right. \\
\left.\mathrm{g}^{-1}\right)\end{array}$ \\
\hline Témoin & $\begin{array}{l}0,50 \pm 0 \\
02 a\end{array}$ & $\begin{array}{l}0,09 \pm \\
0,01 \mathrm{a}\end{array}$ & $\begin{array}{l}5,68 \pm \\
0,62 a\end{array}$ & $\begin{array}{l}5,15 \pm 0, \\
06 \mathbf{a}\end{array}$ & $\begin{array}{l}0,12 \pm 0 \\
06 \mathbf{a}\end{array}$ & $\begin{array}{l}4,98 \pm \\
0,15 a\end{array}$ & $\begin{array}{l}0,23 \pm \\
0,01 \mathrm{a}\end{array}$ & $\begin{array}{l}0,20 \pm \\
0,01 a\end{array}$ & $\begin{array}{l}0,04 \pm 0,0 \\
1 \mathbf{a}\end{array}$ & $\begin{array}{l}0,04 \pm 0, \\
01 \mathrm{a}\end{array}$ \\
\hline $\begin{array}{l}\text { Fumier de } \\
\text { bovin }\end{array}$ & $\begin{array}{l}2,20 \pm 0, \\
04 c\end{array}$ & $\begin{array}{l}0,26 \pm \\
0,01 \mathrm{c}\end{array}$ & $\begin{array}{l}8,46 \pm \\
0,1 \mathrm{~b}\end{array}$ & $\begin{array}{l}6,50 \pm 0, \\
03 \mathrm{~b}\end{array}$ & $\begin{array}{l}0,13 \pm 0, \\
06 \mathbf{a}\end{array}$ & $\begin{array}{l}6,45 \pm \\
0,02 b\end{array}$ & $\begin{array}{l}0,66 \pm \\
0,02 c\end{array}$ & $\begin{array}{l}0,36 \pm \\
0,01 \mathrm{c}\end{array}$ & $\begin{array}{l}0,46 \pm 0,0 \\
1 \mathrm{c}\end{array}$ & $\begin{array}{l}0,26 \pm 0, \\
02 c\end{array}$ \\
\hline Compost & $\begin{array}{l}1,87 \pm 0 \\
05 b\end{array}$ & $\begin{array}{l}0,16 \pm \\
0,01 b\end{array}$ & $\begin{array}{l}12,20 \\
\pm 0,81 \\
\text { c }\end{array}$ & $\begin{array}{l}6,20 \pm 0 \\
07 \mathrm{~b}\end{array}$ & $\begin{array}{l}0,16 \pm 0 \\
01 a\end{array}$ & $\begin{array}{l}6,20 \pm \\
0,05 b\end{array}$ & $\begin{array}{l}0,35 \pm \\
0,02 b\end{array}$ & $\begin{array}{l}0,28 \pm \\
0,01 \mathbf{b}\end{array}$ & $\begin{array}{l}0,27 \pm 0,0 \\
2 \mathbf{b}\end{array}$ & $\begin{array}{l}0,47 \pm 0 \\
02 \mathbf{b}\end{array}$ \\
\hline$F$ & 466,84 & 98,11 & 28,82 & 139,15 & 3,48 & 67,84 & 165,64 & 23,47 & 177,79 & 23,04 \\
\hline$P$ & 0,000 & 0,000 & 0,000 & 0,000 & 0,075 & 0,000 & 0,000 & 0,000 & 0,000 & 0,000 \\
\hline
\end{tabular}

P.ass : Phosphore assimilable

Les moyennes d'une même colonne suivies de lettres différentes sont significativement différentes au seuil de $5 \%$.

Effets des amendements organiques utilisés sur la productivité du sol : Le détail des biomasses fraîches des laitues produites est synthétisé dans le tableau 3. La figure 2 montre, quant à elle, l'évolution de ces biomasses au fil du temps. II ressort que la biomasse issue du traitement témoin s'est accrue légèrement du cycle 1 au cycle $2(0,52 \pm 0,01$ à $0,67 \pm 0,03$ t/ha) avant de régresser jusqu'à $0,45 \pm 0,03$ tha au cycle 3 . La biomasse moyenne résultante est de $0,55 \pm 0,07$ t/ha. Cette valeur est significativement différente des biomasses moyennes enregistrées sur les parcelles amendées avec le fumier d'une part $(2,2 \pm 0,15$ tha) et le compost d'autre part $(2,12 \pm 0,19$ t/ha $)(P<0,05)$, qui, elles ne présentent ainsi aucune différence significative. Toutefois, ces deux biomasses ont connu une croissance régulière avec le temps, qui était plus accentuée en présence du fumier. En effet, à la fin du cycle 1, c'est le compost qui a permis une meilleure productivité du sol. $\bar{A}$ la fin des cycles 2 et 3 par contre, l'effet du fumier avait supplanté celui du compost, de telle sorte que les biomasses obtenues à la fin de ces cycles étaient plus importantes avec le fumier de bovin.

Tableau 3 : Biomasses fraîches de laitue produites par cycle de culture en fonction des traitements

\begin{tabular}{l|l|l|l|l}
\hline & \multicolumn{4}{|c}{ Biomasses produites (t/ha) } \\
\cline { 2 - 5 } & Cycle 1 & Cycle 2 & Cycle 3 & Moyenne \\
\hline Témoin & $0,52 \pm 0,01$ & $0,67 \pm 0,03$ & $0,45 \pm 0,03$ & $0,55 \pm 0,07 \mathbf{a}$ \\
\hline Fumier de bovin & $0,33 \pm 0,01$ & $2,69 \pm 0,04$ & $3,78 \pm 0,12$ & $2,2 \pm 0,15 \mathbf{b}$ \\
\hline Compost & $1,95 \pm 0,03$ & $2,17 \pm 0,03$ & $2,24 \pm 0,02$ & $2,12 \pm 0,19 \mathbf{b}$ \\
\hline
\end{tabular}

Les moyennes suivies de lettres différentes sont significativement différentes au seuil de $5 \%$. 


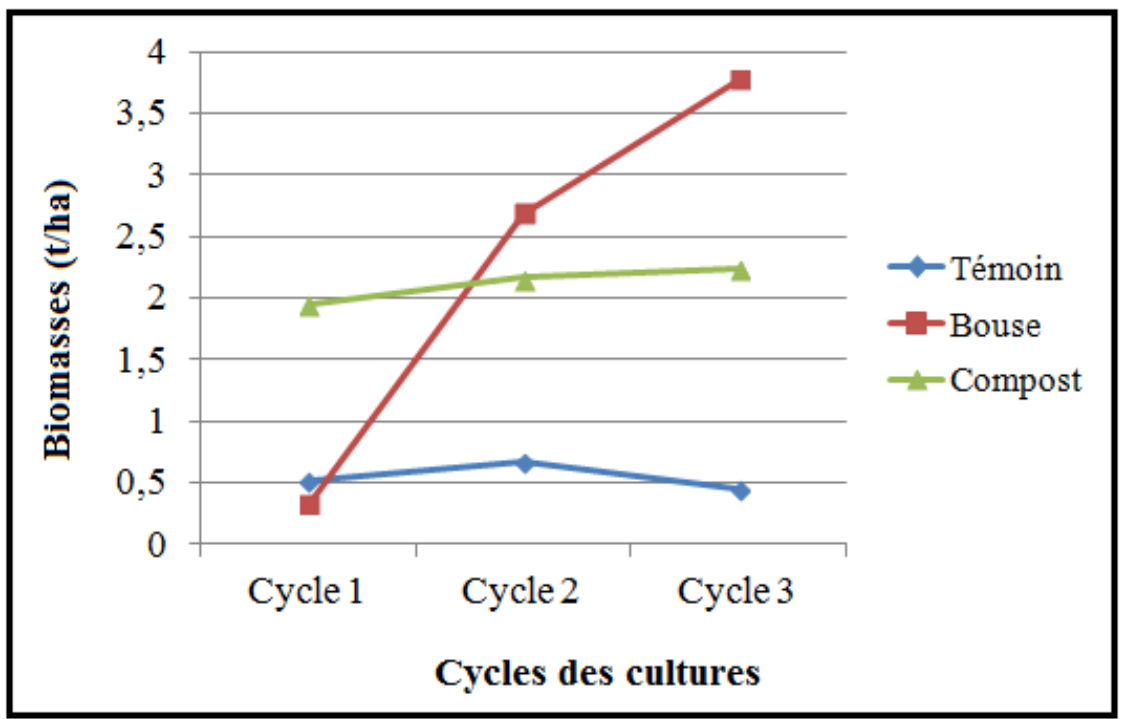

Figure 2 : Évolution des biomasses produites en fonction des traitements et du temps.

\section{DISCUSSION}

Le sol qui a servi de cadre à la réalisation de cette expérimentation est peu fertile à l'image des sols sableux de Côte d'Ivoire en général (Dabin et al., 1960). En effet, sa faible teneur en matière organique, en phosphore, en azote et en potassium le rend peu propice à la plupart des cultures, encore que les risques de sécheresse y soient élevés du fait de faibles teneurs en argiles (moins de $10 \%$ ) couplées à des teneurs très élevées en sables (plus de $80 \%$ ) (Zro et al., 2016). L'application à ce sol des amendements organiques a abouti en fin d'essai à y observer une augmentation du stock de carbone. En conséquence, la CEC, le niveau de phosphore et surtout les niveaux des bases échangeables (potassium, magnésium, calcium et sodium) ont aussi augmenté en général de manière très significative. La neutralisation de l'acidité du sol qui est survenue traduit l'importance des quantités des bases échangeables qui ont été libérées dans le sol. En fait, l'augmentation du stock de carbone dans les sols sableux sous l'effet des amendements organiques a déjà été signalée par plusieurs auteurs dont Delas and Molot (1983), Batamoussi et al. (2016) et Dabre et al. (2017). C'est la preuve que ces types de sol, compte tenu de leur pauvreté généralisée en matières organiques, sont plus sensibles que les autres sols à l'effet des amendements organiques. L'étude a montré également que des deux amendements organiques utilisés, les caractéristiques agronomiques du fumier de bovin sont les meilleures. Ce constat est conforme aux situations habituelles. En effet, Weill and Duval (2009) évoquent la difficulté pour prévoir la valeur fertilisante des composts vu que cette valeur, au contraire de celle des fumiers, dépend des conditions de climat (sécheresse) et de sol (mauvais drainage et $\mathrm{pH}$ bas) peu favorables. Aussi, Tiquia et al. (1997) qui ont apporté un compost immature dans un sol, ont-ils abouti à des effets négatifs sur la germination, la croissance et le développement des plantes. Ici, le fait que le compost utilisé ne soit pas fabriqué dans des conditions optimales devrait être considéré aussi. En effet, ce compost est issu d'un compostage naturel à l'air libre dans des conditions non maîtrisées. Il a été donc exposé pendant longtemps aux intempéries (les pluies et les vents essentiellement) qui ont dû lui fait perdre par lessivage une grande partie de ses constituants minéraux et organiques, principalement l'azote. Cet élément étant l'élément primordial pour le développement des végétaux en général, provoque un retard de croissance chez les cultures lorsque celles-ci en sont carencées (Williams and Haynes, 1995). Sa disponibilité pour les plantes peut être appréciée rapidement par le rapport $\mathrm{C} / \mathrm{N}$ du sol : plus ce rapport est élevé, moins l'azote est disponible (Weill and Duval, 2009). Ainsi, dans la présente étude, le sol témoin qui est était moins fourni en azote était paradoxalement, au vu de son rapport $\mathrm{C} / \mathrm{N}$, le sol où l'azote était plus disponible. Cette teneur d'azote déjà infime pour la culture de laitue mise en place, a été très vite épuisée, d'où la plus faible biomasse enregistrée sur ce sol et la diminution de cette biomasse au fil du temps. Au niveau des sols amendés, le compost, de par son rapport $\mathrm{C} / \mathrm{N}$ plus faible que celui du fumier, aurait au début de l'expérimentation, favorisé la libération de l'azote dans le sol plus rapidement que le fumier. II en est résulté que le sol qui l'a reçu a produit la 
plus grande biomasse à la fin du cycle 1. Cependant, malgré que cette biomasse se soit accrue régulièrement, elle est apparue à la fin des cycles 2 et 3 moins importante que la biomasse enregistrée sur le sol ayant reçu le fumier de bovin. Même si cette différence était non significative, elle traduit non seulement la manifestation de l'effet cumulatif du fumier de bovin, mais aussi, une efficience agronomique du fumier de bovin plus

\section{CONCLUSION}

L'étude entreprise sur l'efficacité du fumier de bovin et du compost d'ordures ménagères en tant qu'amendements des sols sableux a abouti après application d'une même dose de ces amendements à une augmentation du stock de carbone du sol. Par conséquent, la CEC et les niveaux de phosphore, potassium et magnésium échangeables, calcium et sodium ainsi que le $\mathrm{pH}$ du sol ont été améliorés de manière significative. De ce fait, la productivité du sol a été également améliorée de manière

\section{REMERCIEMENTS}

L'équipe de recherche en gestion durable des sols de I'Université Jean Lorougnon Guédé remercie les acteurs du maraîchage de la ville de Daloa pour leur collaboration

\section{RÉFÉRENCES}

Avenard JM: 1971. Aspect de la géomorphologie in : Milieu naturel de Côte d'Ivoire. Mémoire ORSTOM, Paris, France, 50, pp. 8-73.

Batamoussi HM, Tovihoudji GP, Tokore OM, Boulga J et Essegnon IM: 2016. Effet des engrais organiques sur la croissance et le rendement de deux variétés de tomate (Lycopersicum esculentum) dans la commune de Parakou (Nord Bénin). International Journal of Innovation and Scientific Research, Vol. 24, № 1, pp. 8694.

Brou YT : 2005. Climat, mutations socio-économiques et paysages en Côte d'lvoire. Mémoire de synthèse des activités scientifiques. Habilitation à Diriger des Recherches, Université des Sciences et Technologies de Lille, France, 212 p.

CEAEQ: 2005. Détermination du $\mathrm{pH}$ : méthode électrométrique. MA. 100-pH 1.1, Rév. 3, Ministère du Développement durable, de l'Environnement, de la Faune et des Parcs du Québec, 11 pages.

Chabalier PF, Van de Kerchove V. et Saint Macary H: 2006. Guide de la fertilisation organique à la Reunion. CIRAD, 166 p. intéressante que celle du compost. Cette analyse est corroborée par le rapport $\mathrm{C} / \mathrm{N}$ du sol amendé avec le fumier avéré en fin d'expérimentation plus faible que celui du sol qui a reçu le compost. II est donc clair que si d'autres cycles de culture de laitue avaient suivi les trois cycles qui ont été réalisés, les différences entre les deux traitements à base d'amendements seraient de plus en plus significatives.

significative. Toutefois, le fumier de bovin qui a montré une valeur fertilisante plus intéressante et qui, par ailleurs, a permis une meilleure productivité du sol, est plus adaptée à la fertilisation des sols sableux dans les conditions de cette expérimentation. Cependant, les relatives moins bonnes caractéristiques agronomiques affichées ici par le compost peuvent être corrigées si l'on prend soin de trier les ordures avant de procéder à leur compostage à l'abri des intempéries.

lors de cette étude. Nous voudrions également remercier toute la gouvernance de l'Université Jean Lorougnon Guédé (Daloa, Côte d'Ivoire).

Dabin B, Leneuf N. et Riou G: 1960. Carte pédologique de la Côte d'lvoire au $1 / 2.000 .000$. Notice explicative. ORSTOM, $39 p$.

Dabre A, Hien E, Der Some et Drevon JJ: 2017. Effets d'amendements organiques et phosphatés sous zaï sur les propriétés chimiques et biologiques du sol et la qualité de la matière organique en zone soudano-sahélienne du Burkina Faso. International Journal of Biological and Chemical Sciences, 11(1): 473-487.

Delas J. et Molot C: 1983 . Effet de divers amendements organiques sur les rendements du maiis et de la pomme de terre cultivés en sol sableux. Agronomie, 3 (1), pp.19-26.

Fondio L, Kouamé C, Djidji AH et Traoré D: 2011. Caractérisation des systèmes de culture intégrant le gombo dans le maraîchage urbain et périurbain de Bouaké dans le Centre de la Côte d'Ivoire. International Journal of Biological and Chemical Sciences, 5 (3):1178-1189.

Griffon M, Cleaver K, Freud E.H. et EH Freud CM : 1993. Quelles stratégies pour les agricultures en Afrique subsaharienne ? CIRAD, Coll. Notes et Documents, $16: 62 \mathrm{p}$. 
Jouve $P$ : 2011. Enjeux liés à la production, Grain de sel $\mathrm{N}^{0} 54-56$, pp. 23-24.

Milleville P. et Serpante G: 1994. Intensification et durabilité des systèmes agricoles en Afrique soudano-sahélienne. In: Promotion de systèmes agricoles durables dans les pays d'Afrique soudano-sahélienne. Wageningen: CTA, 33-45.

Pétard J: 1993. Les méthodes d'analyse. Tome 1: Analyses de sols, Laboratoire commun d'analyses $\mathrm{N}^{\circ} 5,200 \mathrm{p}$.

Temgoua E, Tsafack NH, Ngnikam E, Gouana TR and Dongmo ZRG : 2017. Fertilisation du maïs (Zea mays $L$.) à base d'urines humaines hygiénisées dans un oxisol de l'Ouest Cameroun. International Journal of Biological and Chemical Sciences, 11(5): 2071-2081.

Tiquia SM, Tam NFY and Hodgkiss IJ: 1997. Effects of turning frequency on composting of spent pig manure sawdust litter. Bioresource Technology, 62: 37-42.

Weill A et Duval J: 2009. Guide de gestion globale de la ferme maraîchère biologique et diversifiée. Equiterre, Module 7, Chapitre 12, $19 \mathrm{p}$.
Williams PH and Haynes RJ : 1995. Effect of sheep, deer and cattle dung on herbage production and soil nutrient content. Grass Forage Sci., 50, 263271.

Zro FGB, Guéi AM, Konaté Z, Soro D, Bakayoko S and Yao-Kouamé A: 2017. Impacts of soil fertilization based on fresh bovine dung on the quality of lettuce (Lactuca sativa L.) produced in the market agrosystems of the town of Daloa (Côte d'Ivoire). International Journal of Development Research, Vol. 07, pp.1293012939.

Zro FGB, Guéi AM, Nangah YK, Soro D and Bakayoko S: 2016. Statistical approach to the analysis of the variability and fertility of vegetable soils of Daloa (Côte d'Ivoire). African Journal of Soil Science, Vol. 4 (4), pp. 328-338.

Zro FGB, Guéi AM, Nangah YK and Yao-Kouamé A: 2018. Impacts of household waste compost formed in public garbage dump on the organomineral status and productivity of a sandy soil. International Journal of Engineering and Applied Sciences, Volume 5, pp. 1-5. 